\title{
Investigation of the effect of phlomisoside $F$ on complete Freund's adjuvant-induced arthritis
}

\author{
XIUYING ZHANG ${ }^{1}$, YANFENG DONG ${ }^{2}$, HANYU DONG $^{3}$, WEN ZHANG $^{4}$ and FANG LI $^{5}$ \\ ${ }^{1}$ Rheumatism Immunity Branch, Zibo Central Hospital, Zibo, Shandong 255036; ${ }^{2}$ Department of Cardiovasology, \\ The People's Hospital of Zhangdian, Zibo, Shandong 255000; ${ }^{3}$ Taishan Medical College, Taian, Shandong 271016; \\ ${ }^{4}$ Central Lab of Zibo Central Hospital, Zibo, Shandong 255036; ${ }^{5}$ Rheumatism Immunity Branch, \\ Taian Central Hospital, Taian, Shandong 271000, P.R. China
}

Received July 5, 2015; Accepted September 27, 2016

DOI: $10.3892 /$ etm.2016.3995

\begin{abstract}
Phlomis younghusbandii Mukerjee (Labiatae) has been reported to be effective in the treatment of rheumatoid arthritis (RA). In the present study, the anti-inflammatory and anti-arthritic effects of phlomisoside $\mathrm{F}(\mathrm{PF})$, isolated from $P$. younghusbandii Mukerjee (Labiatae), were investigated in male Wistar rats subjected to carrageen-induced paw edema and complete Freund's adjuvant (CFA)-induced arthritis. Arthritis scores were evaluated by a 5-point ordinal scale (scores 0-4). Expression levels of TNF- $\alpha$, IL-1 $\beta$, IL-6, IL-10, COX-2 and 5-LOX were determined via ELISA and western blot assays. Subsequent to establishing the edema and arthritis models, oral administration of PF $(5,10$ and $20 \mathrm{mg} / \mathrm{kg}$ ) significantly inhibited mean edema rate, compared with the control group in carrageenan-induced paw edema assay. In addition, administration of $\mathrm{PF}$ (5, 10 and $20 \mathrm{mg} / \mathrm{kg} /$ day $)$ for 28 days markedly exhibited an anti-arthritic activity by offsetting the body weight loss, inhibiting the paw edema, reducing the arthritis scores and the indices of thymus and spleen, inhibiting the expression levels of TNF- $\alpha$, IL-1 $\beta$, IL-6, COX-2 and 5-LOX, and increasing the expression of IL-10, when compared with the respective control group in CFA-induced arthritis assay. In conclusion, $\mathrm{PF}$ is a valuable anti-arthritic constituent of $P$. younghusbandii, and the present study results suggest that this herb may be used in the treatment of RA.
\end{abstract}

\section{Introduction}

Rheumatoid arthritis (RA) is a chronic, inflammatory, systemic autoimmune disease (1) that is characterized by synovial membrane inflammation, swelling, autoantibody production,

Correspondence to: Dr Fang Li, Rheumatism Immunity Branch, Tiain Central Hospital, 29 Longtan Road, Taian, Shandong 271000, P.R. China

E-mail: fli_2015@163.com

Key words: Phlomis younghusbandii, complete Freund's adjuvant, adjuvant arthritis, anti-inflammatory activity, anti-arthritic activity cartilage and bone destruction (2). RA more commonly affects middle-aged females, and is associated with systemic complications, disability, early mortality and socioeconomic costs (3).

Currently, common agents administered for symptom relief and delay of RA progression mainly include nonsteroidal anti-inflammatory drugs (NSAIDs) (4), disease-modifying antirheumatic drugs (5), hormone drugs (such as glucocorticoids) (6) and biological agents $(7,8)$. Furthermore, combination of these drugs is the major strategy for the treatment of RA $(9,10)$. However, these drugs are expensive and result in side effects after long-term use, such as NSAID-induced small bowel injury (11) or glucocorticoid-induced non-serious infections (12), thus limiting their application.

Due to the advantages of traditional Chinese medicine (TCM) in the treatment of various diseases and reduced incidence of side effects, they have been widely used for the treatment of RA. Monomeric compounds or TCM herbal prescriptions can be used for the treatment of RA. Compounds that have been reported to have anti-arthritic effects include: Alkaloids, such as tetramethylpyrazine (13), sinomenine (14) and brucine (15); glycosides, including tripterygium polyglycoside (16), total glucosides of peony (17) and madecassoside (18); lactones, such as artesunate (19) and artemether (20); quinines, such as shikonin (21); and various TCM prescriptions, including Sanwushaoxie decoction (22) and Qingluo Tongbi Granula (23).

Phlomis Radix, obtained from the plant Phlomis younghusbandii Mukerjee (family Labiatae), is commonly used for the treatment of cold, cough, sore throat, pyogenic infections, RA, pneumonia and bronchitis (24-27). The present study was designed to investigate the anti-arthritic effect and underlying mechanism of phlomisoside $\mathrm{F}(\mathrm{PF})$, which is isolated from Phlomis Radix, in rats subjected to carrageen-induced paw edema and complete Freund's adjuvant (CFA)-induced arthritis.

\section{Materials and methods}

Animals. A total of 210 male Wistar rats (age, 2 months; weight, 150-200 g) were used to evaluate the anti-inflammatory and anti-arthritic effects of PF in the present study. 
All animals were obtained from the Experimental Animal Center of the Second Military Medical University (Shanghai, China). The animals were kept at standard laboratory conditions (temperature, $25 \pm 1^{\circ} \mathrm{C} ; 12 \mathrm{~h} \mathrm{light/dark}$ cycle), and had free access to standard food and water. All experiments were performed in accordance with the international guidelines for the care and use of laboratory animals. The experimental protocols were approved by the Animal Care and Use Committee of Taian Central Hospital (Taian, China; protocol no. CHTA2014021).

Plant material. Phlomis Radix, the dry root of Phlomis younghusbandii, was collected from Changdu (Xizang, China) in 2014. The original plant was identified by Dr Hanyu Dong (Taishan Medical College). A voucher specimen (no. 201423/CHTA) was stored at the Department of Pharmacy of Taian Central Hospital for future reference.

Chemicals and reagents. Silica gel (200-300 mesh) was purchased from Qingdao Haiyang Chemical Co., Ltd. (Qingdao, China). High-performance liquid chromatography (HPLC) grade methanol, and analytical reagents ethyl acetate and ethanol were obtained from Sinopharm Chemical Reagent Co., Ltd. (Shanghai, China). CFA was obtained from Sigma-Aldrich (St. Louis, MO, USA). Tumor necrosis factor (TNF)- $\alpha$ (RTA00), interleukin (IL)-1 $\beta$ (RLB00), IL-6 (R6000B) and IL-10 (R1000) ELISA kits were obtained from R\&D System (Minneapolis, MN, USA). Primary antibodies for cyclooxygenase-2 (COX-2; 4842) and 5-lipoxygenase (5-LOX; 3289) were obtained from Cell Signaling Technology, Inc. (Beverly, MA, USA); $\beta$-actin (AF0003) was purchased from Beyotime Institute of Biotechnology (Haimen, China). Horseradish peroxidase (HRP)-conjugated goat anti-rabbit antibody was obtained from Abcam (Cambridge, UK).

Preparation of PF. The dried powder of Phlomis Radix (30 kg) was extracted twice by refluxing with $95 \%$ ethanol. Next, the ethanol extract was concentrated under reduced pressure to produce a crude extract, which was then suspended in water and successfully partitioned with ethyl acetate (EtOAc). The EtOAc fraction was eluted with trichloromethane-methanol (14:1, 8:1 and 5:1) to obtain PF on a silica-gel column (200-300 mesh). The identification and purity of PF were analyzed on a Bruker Avance III HD nuclear magnetic resonance (NMR) spectrometer (Bruker, Bremen, Germany) and Agilent 1290 Infinity LC and HPLC system (Agilent Technologies, Inc., Santa Clara, CA, USA), respectively. The chromatographic column used was a Tianhe $\mathrm{C}_{18}$ column $(250 \times 4.6 \mathrm{~mm}, 5 \mu \mathrm{m})$ from Tianhe Heng Kang Technology Co., Ltd., (Beijing, China). The mobile phase was methanol-water (70:30), and the detection wavelength was $248 \mathrm{~nm}$.

Acute toxicity assay. In order to determine the concentration at which PF induced acute toxicity to rats, they were treated with various concentrations of PF. Rats were randomly divided into 10 groups ( $\mathrm{n}=10$ per group), and were orally administered with $0,20,40,60,80,100,120,140,160$ or $180 \mathrm{mg} / \mathrm{kg} \mathrm{PF}$, respectively (27). The acute toxicity test suggested that PF treatment up to the concentration of $180 \mathrm{mg} / \mathrm{kg}$ did not result in rat mortality within the observation period.
Carrageenan-induced paw edema assay. The preliminary anti-inflammatory effect of PF was investigated by a carrageenan-induced paw edema assay. Rats were randomly divided into five groups (10 rats in each group), including the normal control, diclofenac sodium (serving as a positive control) and three PF groups. The control group was orally treated with normal saline $(10 \mathrm{ml} / \mathrm{kg})$, and the diclofenac sodium group was treated with isopycnic diclofenac sodium (10 $\mathrm{mg} / \mathrm{kg}$ ), which is a known anti-inflammatory (28), dissolved in normal saline. The three PF treatment groups were administered with isopycnic PF at a concentration of 5,10 or $20 \mathrm{mg} / \mathrm{kg}$, dissolved in normal saline. At $30 \mathrm{~min}$ after administration, $1 \%$ (w/v) carrageenan (50 $\mu \mathrm{l}$; Sigma-Aldrich) was subcutaneously injected into the subplantar tissue of the right hind paw, and then the paw volumes of rats were measured with a plethysmograph after $0.5,1,2$ and $4 \mathrm{~h}$. Edema rate and inhibition rate were calculated to evaluate the anti-inflammatory effect of $\mathrm{PF}$, using the following formulas: Edema rate $(\%)=(\mathrm{A}-\mathrm{B}) / \mathrm{B}$, in which $\mathrm{A}$ and $\mathrm{B}$ represented the paw volume of the right hind paw and the left hind paw, respectively; inhibition rate $(\%)=(\mathrm{C}-\mathrm{D}) / \mathrm{C} x 100$, in which $\mathrm{C}$ and $\mathrm{D}$ represented the average edema rates of the control group and the diclofenac sodium and PF groups, respectively.

Adjuvant arthritis (AA) model and treatment. Prior to the experiment, rats were randomly divided into six groups (10 rats in each group), including the normal, AA model, methotrexate (MTX; serving as a positive control; Sigma-Aldrich) and three PF groups. CFA was prepared by suspending heat-killed Bacillus Calmette-Guérin (YuanMu Biological Technology Co., Ltd., (Shanghai, China) in liquid paraffin at $20 \mathrm{mg} / \mathrm{ml}$ (29). With the exception of the normal group, the AA animal model was established in all groups by intradermal injection of $0.1 \mathrm{ml} \mathrm{CFA}$ into the left hind metatarsal foot pad of rats, which induced inflammation $(30,31)$. Subsequently, the MTX group was orally treated with $3 \mathrm{mg} / \mathrm{kg}$ MTX twice a week for 28 days. The three PF groups were orally treated at doses of 5,10 or $20 \mathrm{mg} / \mathrm{kg} /$ day for 28 days. In addition, the normal and AA model groups were orally treated with isopycnic normal saline for 28 days.

Evaluation of arthritis. Changes in the body weight of rats, hind paw swelling and arthritis scores were used to evaluate the anti-arthritic effect of PF in the CFA-induced AA model. Body weight changes in the rats were measured on days $7,14,21$ and 28 of the experiments. The paw volumes of hind paw swelling were measured with a plethysmograph every 7 days. For evaluating signs of arthritis, the AA rats were assessed every 4 days for 28 days using a 5-point ordinal scale, which is a pre-established, widely used scoring system developed to evaluate the severity of adjuvant arthritis $(32,33)$. The rat paws were examined and scored for severity, swelling, erythema and induration by a 5-point ordinal scale (scores 0-4) as follows: 0 , unaffected; 1, one type of joint affected; 2, two types of joint affected; 3, three types of joint affected; 4, three types of joint affected plus maximal erythema and swelling. The maximum score of every arthritic rat was 8 (4 points $\mathrm{x} 2$ hind paws).

Thymus and spleen assays. At day 28 after CFA-induced AA, all rats were directly sacrificed via intraperitoneal injection 
Table I. Anti-inflammatory effect of PF in carrageenan-induced paw edema assay.

\begin{tabular}{lcccc}
\hline & \multicolumn{4}{c}{ Edema rate (inhibition rate; \%) } \\
\cline { 2 - 5 } Groups & $0.5 \mathrm{~h}$ & $1 \mathrm{~h}$ & $2 \mathrm{~h}$ & $4 \mathrm{~h}$ \\
\hline Normal saline $(10 \mathrm{ml} / \mathrm{kg})$ & $24.96 \pm 5.95$ & $43.78 \pm 12.63$ & $55.09 \pm 8.93$ & $90.09 \pm 16.02$ \\
Diclofenac sodium $(10 \mathrm{mg} / \mathrm{kg})$ & $13.15 \pm 7.24^{\mathrm{b}}$ & $22.56 \pm 8.25^{\mathrm{b}}$ & $29.35 \pm 9.38^{\mathrm{b}}$ & $44.53 \pm 12.21^{\mathrm{b}}$ \\
& $(47.32)$ & $(48.47)$ & $(53.27)$ & $(50.57)$ \\
$5 \mathrm{mg} / \mathrm{kg}$ PF & $18.64 \pm 6.28^{\mathrm{a}}$ & $34.52 \pm 9.63^{\mathrm{a}}$ & $44.28 \pm 6.03^{\mathrm{a}}$ & $69.17 \pm 16.46^{\mathrm{a}}$ \\
& $(25.32)$ & $(21.15)$ & $(19.62)$ & $(23.22)$ \\
$10 \mathrm{mg} / \mathrm{kg}$ PF & $14.68 \pm 5.68^{\mathrm{b}}$ & $23.64 \pm 8.885^{\mathrm{b}}$ & $34.31 \pm 10.01^{\mathrm{b}}$ & $52.49 \pm 13.42^{\mathrm{b}}$ \\
& $(41.19)$ & $(46.00)$ & $(37.72)$ & $(41.74)$ \\
$20 \mathrm{mg} / \mathrm{kg}$ PF & $13.19 \pm 6.51^{\mathrm{b}}$ & $19.43 \pm 9.02^{\mathrm{b}}$ & $30.14 \pm 9.23^{\mathrm{b}}$ & $49.18 \pm 13.15^{\mathrm{b}}$ \\
& $(47.16)$ & $(55.62)$ & $(45.29)$ & $(45.41)$ \\
\hline
\end{tabular}

${ }^{\mathrm{a}} \mathrm{P}<0.05$ and ${ }^{\mathrm{b}} \mathrm{P}<0.01$, vs. normal saline group. $\mathrm{PF}$, phlomisoside $\mathrm{F}$.

of $40 \mathrm{mg} / \mathrm{kg}$ pentobarbital (Sigma-Aldrich), and the entire thymus and spleen were removed immediately and weighed. The evaluation indices of thymus and spleen were evaluated according to the ratio of the thymus and spleen wet weight (mg) over the body weight of rats $(\mathrm{g})(34,35)$.

Determination of TNF- $\alpha, I L-1 \beta, I L-6$ and IL-10 concentrations in the serum. Rat serum was obtained by collecting and clotting blood samples for $30 \mathrm{~min}$ at room temperature and then freezing at $-20^{\circ} \mathrm{C}$ until needed $(36,37)$. The protein concentration of TNF- $\alpha$, IL-1 $\beta$, IL- 6 and IL-10 in the serum was determined using the corresponding ELISA kits, according to the manufacturer's instructions, and an FLx800 Fluorescence microplate reader (BioTek Instruments, Inc., Winooski, VT, USA).

Determination of COX-2 and 5-LOX levels in peripheral blood mononuclear cells (PBMCs). Blood samples were used to obtain PBMCs by Ficoll-Paque density gradient centrifugation, as previously described $(31,38)$. The total proteins of PBMC were extracted using cell lysis buffer, ultrasound and centrifugation at $12,000 \times \mathrm{g}$ for $15 \mathrm{~min}$ at $4^{\circ} \mathrm{C}$. Their concentration was measured with the Enhanced BCA Protein Assay reagent (Beyotime Institute of Biotechnology). Next, total proteins $(40 \mu \mathrm{g})$ were separated by $10 \%$ SDS-PAGE, transferred to polyvinylidene difluoride (PVDF) membranes. After the PVDF membranes were blocked with 5\% skim milk for $2 \mathrm{~h}$ at room temperature, they were incubated with homologous primary antibodies against COX-2, 5-LOX and $\beta$-actin overnight at $4^{\circ} \mathrm{C}$ (dilution, 1:1,000). Subsequently, the membrane was washed with Tris-buffered saline-Tween 20 (TBS-T), and then further incubated with HRP-conjugated goat anti-rabbit antibodies in TBS-T. The COX-2 and 5-LOX expression levels were detected by chemiluminescence with BeyoECL Plus reagents (P0018; Beyotime Institute of Biotechnology). In order to assess protein loading, $\beta$-actin was selected as an internal reference.

Statistical analysis. All data are presented as the mean \pm standard deviation and relative results were analyzed by SPSS

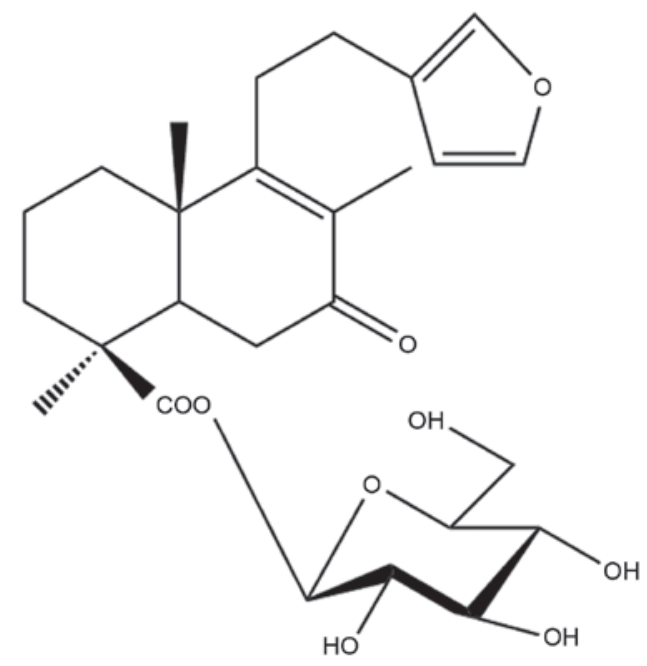

Figure 1. Chemical structure of phlomisoside F.

version 21.0 statistical software (IBM Corp., Armonk, NY, USA). One-way analysis of variance and a nonparametric test $\left(\chi^{2}\right.$ test) were used to evaluate whether the difference between the various experiments was statistically significant, which was indicate by P-values of $<0.05$.

\section{Results}

Identification and purity of $P F$. The target analyte was identified as PF according to the NMR results and compared with the existing literature (39). The chemical structure of PF is shown in Fig. 1. In addition, the purity of PF was determined by the HPLC area normalization method and was found to be $>93 \%$.

Anti-inflammatory activity of $P F$ in carrageenan-induced paw edema assay. Inflammation is the common process of arthritis, and thus a carrageenan-induced paw edema assay was used to investigate the preliminary anti-inflammatory activity of PF in the present study. As shown in Table I, treatment with PF (5, 10 and $20 \mathrm{mg} / \mathrm{kg}$ ) resulted in significant 




Figure 2. Effect of PF on changes in the body weight of CFA-injected rats. ${ }^{* *} \mathrm{P}<0.01$ vs. normal group. PF, phlomisoside F; CFA, complete Freund's adjuvant; AA, adjuvant arthritis; MTX, methotrexate.

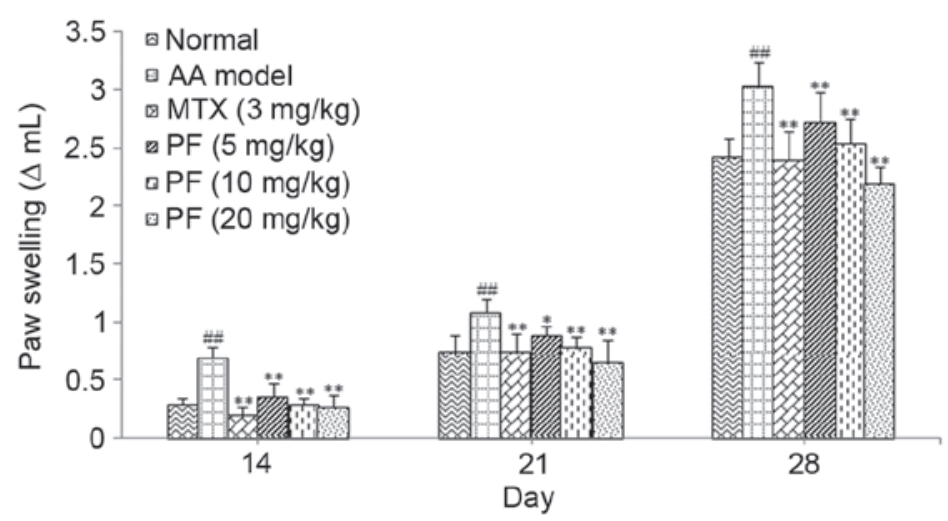

Figure 3. Effect of $\mathrm{PF}$ on paw swelling of CFA-injected rats. ${ }^{\# \#} \mathrm{P}<0.01$, vs. normal group; ${ }^{*} \mathrm{P}<0.05$ and ${ }^{* *} \mathrm{P}<0.01$, vs. AA model group. $\mathrm{PF}$, phlomisoside $\mathrm{F}$; CFA, complete Freund's adjuvant; AA, adjuvant arthritis; MTX, methotrexate.

anti-inflammatory activity $(\mathrm{P}<0.05)$ at $0.5,1,2$ and $4 \mathrm{~h}$ after carrageenan administration, compared with the normal control group (normal saline). Namely, PF may have an anti-arthritic activity, and thus an AA rat model was then used to examine its effect.

Effect of PF on the changes in body weight, paw swelling and arthritis score in the CFA-induced AA model. As depicted in Fig. 2, the body weight increase in the AA model group was slower compared with that in the normal group. In addition, on days 21 and 28, the AA model group exhibited a significant body weight loss $(\mathrm{P}<0.01)$, compared with the normal group. However, the body weight loss of CFA-injected rats was offset after treatment with 5, 10 and $20 \mathrm{mg} / \mathrm{kg} \mathrm{PF}$. Although the recuperative rate of body weight loss in the PF treated groups was lower than in the normal group, it was still higher compared with the model group, with no significant differences observed between the normal and PF groups.

As shown in Fig. 3, the paw swelling volume showed an evident difference from day 14 among the different groups. Compared with the normal group, the paw swelling volume of the model group exhibited a marked $(\mathrm{P}<0.01)$ increase, indicating that the AA animal model was successfully established. Compared with the AA model group, the MTX (3 mg/kg) and $\mathrm{PF}(5,10$ and $20 \mathrm{mg} / \mathrm{kg})$ groups demonstrated significantly inhibited paw swelling in the CFA-injected rats $(\mathrm{P}<0.05)$.
The arthritis scores of the MTX (3 mg/kg) and PF $(5,10$ and $20 \mathrm{mg} / \mathrm{kg}$ ) groups were significantly reduced from day 12 of treatment, compared with those in the AA model group $(\mathrm{P}<0.01$; Fig. 4). These results suggested that $\mathrm{PF}$ exhibited an inhibitory effect on CFA-induced AA by inhibiting inflammatory responses.

Effect of PF on the evaluation indices of the thymus and spleen in the AA model. The indices of the thymus and spleen in the AA model group were significantly increased, compared with the normal group $(\mathrm{P}<0.01$; Fig. 5), suggesting that the model was successfully established. Compared with the AA model group, the indices of the thymus and spleen in the MTX (3 mg/kg) and PF (5, 10 and $20 \mathrm{mg} / \mathrm{kg}$ ) groups were significantly reduced $(\mathrm{P}<0.01$; Fig. 5$)$.

Effect of PF on the expression levels of TNF- $\alpha, I L-1 \beta, I L-6$ and $I L-10$ in the serum of AA model rats. As shown in Fig. 6, the expression levels of TNF- $\alpha$, IL-1 $\beta$, IL- 6 were significantly increased $(\mathrm{P}<0.01)$, whereas IL-10 expression was markedly reduced $(\mathrm{P}<0.01)$, in the AA model group compared with the normal group; this suggested that the model was successfully established. Compared with the AA model group, the MTX $(3 \mathrm{mg} / \mathrm{kg})$ and PF $(5,10$ and $20 \mathrm{mg} / \mathrm{kg}$ ) groups were demonstrated to have evidently inhibited expression levels of TNF- $\alpha$, IL-1 $\beta$ and IL-6, and increased IL-10 expression $(\mathrm{P}<0.05$ 


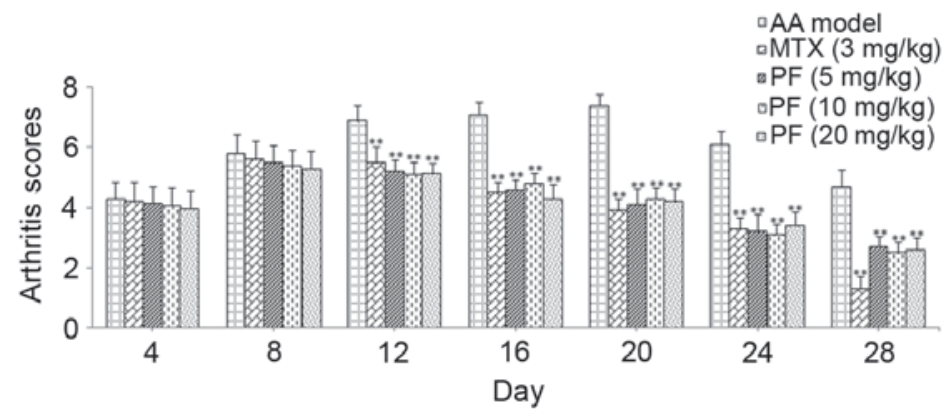

Figure 4. Effects of PF on arthritis scores of CFA-injected rats. " $\mathrm{P}<0.01$ vs. AA model group. PF, phlomisoside F; CFA, complete Freund's adjuvant; AA, adjuvant arthritis; MTX, methotrexate.

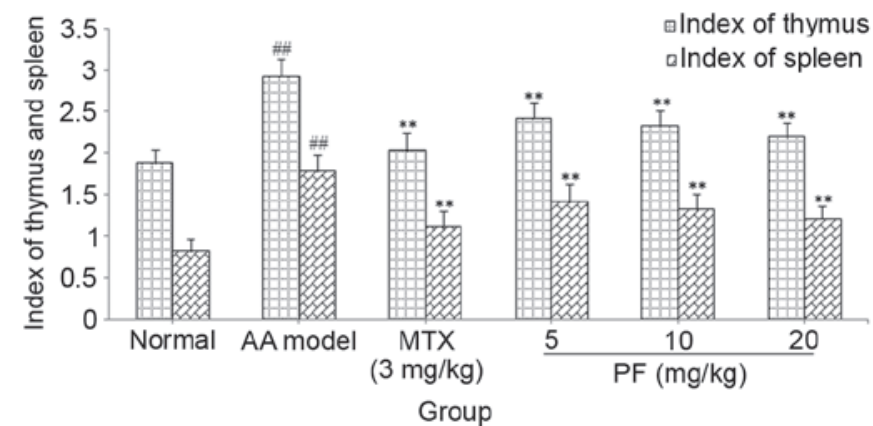

Figure 5. Effects of PF on evaluation indices of thymus and spleen of CFA-injected rats. ${ }^{\# \#} \mathrm{P}<0.01$ vs. normal group; ${ }^{* *} \mathrm{P}<0.01$ vs. AA model group. PF, phlomisoside F; CFA, complete Freund's adjuvant; AA, adjuvant arthritis; MTX, methotrexate


Figure 6. Effects of PF on the expression levels of TNF- $\alpha$, IL-1 $\beta$, IL-6 and IL-10 in the serum of CFA-injected rats. ${ }^{\# \prime} \mathrm{P}<0.01$ vs. normal group; ${ }^{*} \mathrm{P}<0.05$ and ${ }^{* *} \mathrm{P}<0.01$ vs. AA model group. $\mathrm{PF}$, phlomisoside $\mathrm{F}$; TNF, tumor necrosis factor; IL, interleukin; CFA, complete Freund's adjuvant; AA, adjuvant arthritis; MTX, methotrexate.

or $\mathrm{P}<0.01)$. These results indicated that the inhibitory effect of $\mathrm{PF}$ on inflammatory responses may be associated witho downregulating TNF- $\alpha$, IL-1 $\beta$ and IL-6, and upregulating IL-10.
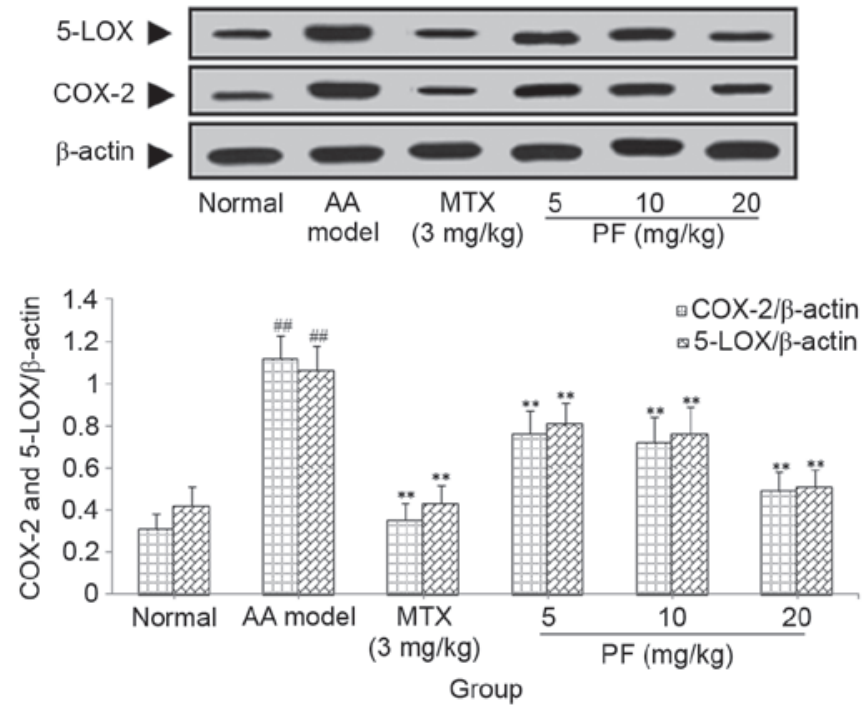

Figure 7. Effects of PF on the expression levels of COX-2 and 5-LOX in the peripheral blood mononuclear cells of CFA-injected rats, as observed by western blot analysis. ${ }^{\# /} \mathrm{P}<0.01$ vs. normal group; ${ }^{* *} \mathrm{P}<0.01$ vs. AA model group. PF, phlomisoside F; COX-2, cyclooxygenase-2; 5-LOX, 5-lipoxygenase; CFA, complete Freund's adjuvant; AA, adjuvant arthritis; MTX, methotrexate.

Effect of PF on the expression levels of COX-2 and 5-LOX in PBMCs. As shown in Fig. 7, the expression levels of COX-2 and 5-LOX in the AA model group were significantly upregulated compared with the normal group $(\mathrm{P}<0.01)$, which suggested that the model was successful. Compared with AA model group, the expression levels of COX-2 and 5-LOX in the MTX $(3 \mathrm{mg} / \mathrm{kg})$ and PF $(5,10$ and $20 \mathrm{mg} / \mathrm{kg}$ ) groups were evidently downregulated $(\mathrm{P}<0.01)$. These results indicated that the inhibitory effect of $\mathrm{PF}$ on inflammatory responses may be associated with downregulating COX-2 and 5-LOX.

\section{Discussion}

In the present study, PF isolated from Phlomis Radix was demonstrated to have anti-inflammatory and anti-arthritic activities as observed by carrageenan-induced paw edema and CFA-induced arthritis assays.

Carrageenan-induced paw edema model is widely used to evaluate the anti-inflammatory activity of drugs $(40,41)$. The results of carrageenan-induced paw edema assay in the current study suggested that $\mathrm{PF}$ treatment $(5,10$ and $20 \mathrm{mg} / \mathrm{kg})$ had an 
evident anti-inflammatory activity at $0.5,1,2$ and $4 \mathrm{~h}(\mathrm{P}<0.05)$. It has been reported that Phlomis Radix can be used to treat RA (26). For instance, Wang et al (27) demonstrated that the methanol extract from the aerial parts of Phlomis younghusbandii has anti-inflammatory and anti-nociceptive effects, and that PF is found in the methanol extract. Inflammation is the key clinical manifestation of arthritis (42), thus, the anti-arthritic activity of PF in the AA model was investigated in the current study in order to determine whether PF is a major anti-arthritic constituent in Phlomis younghusbandii.

The CFA-induced arthritis model is widely used to study the anti-arthritic activity of drugs $(43,44)$. Alterations in the body weight of animals, paw swelling and arthritis scores are common indices used to evaluate the anti-arthritic effect of drugs in the CFA-induced arthritis model. In the present study, CFA-injected rats exhibited a marked weight loss, which was reversed by administration of PF. Weight loss following CFA injection and the reversal by PF may be associated with inflammatory cytokine levels (45), thus, the change in the body weight of rats may be an important index for the evaluation of the anti-arthritic activity of PF. In addition, CFA-injected rats exhibited an evident increase in paw swelling and arthritis scores, which were significantly reversed following the administration of PF.

Thymus and spleen are two key immune organs, and the ratio of their wet weight over the body weight of homologous rats is commonly used to evaluate the immunoregulatory effect of drugs $(34,35)$. The indices of thymus and spleen in CFA-injected rats were evidently increased in the current study, and these were markedly reversed following administration of PF. Therefore, PF may exert it anti-arthritic effect by downregulating indices of the thymus and spleen.

The pro-inflammatory cytokines (such as TNF- $\alpha$, IL- $1 \beta$ and IL-6) and anti-inflammatory cytokines (such as IL-10) are known to serve an important role in inflammation (46-49). ELISA analysis performed in the present study indicated that the concentrations of TNF- $\alpha$, IL- $1 \beta$ and IL- 6 were significantly upregulated, while IL-10 concentration was markedly downregulated, in the serum of CFA-injected rats. This effect of CFA was evidently reversed subsequent to the administration of PF, indicating that PF may obtain its anti-arthritic effect by downregulating TNF- $\alpha$, IL-1 $\beta$ and IL-6, and upregulating IL-10.

COX is a diffusion speed limiting enzyme involved in the synthesis of prostaglandins (PGs). There are two types of COX isozymes: COX-1, which is a housekeeping enzyme used to maintain the body's normal functions; and COX-2, an enzyme induced by traumatic chemical, physical and biological factors, which catalyzes the synthesis of PGs and is involved in the positive inflammatory response (50,51). 5-LOX is another major speed limit enzyme involved in the synthesis of leukotrienes and in the positive inflammatory response induced by arachidonic acid (52). In the current experiments, the expression levels of COX-2 and 5-LOX in the PBMCs of CFA-injected rats were significantly upregulated, which was evidently reversed following the administration of PF. This finding indicated that PF may obtain its anti-arthritic effect by downregulating the expression levels of COX-2 and 5-LOX. Furthermore, according to the effect of the three PF doses $(5,10$ and $20 \mathrm{mg} / \mathrm{kg})$ on all arthritis-associated indices, the anti-arthritic activity of PF may be dose-dependent. According to these results, it can be inferred that PF had an anti-arthritic activity and may be one of the major anti-arthritic constituents of Phlomis younghusbandii.

In conclusion, the present study indicated that PF is a valuable anti-arthritic constituent of Phlomis younghusbandii, suggesting that this herb may be applied to treat RA. PF was found to reverse weight loss, to reduce paw swelling and the indices of thymus and spleen, as well as to reduce the arthritis scores of CFA-injected rats, when compared with AA model rats. The mechanisms of the anti-arthritic effect of PF may be associated with downregulation of pro-inflammatory cytokines (TNF- $\alpha$, IL-1 $\beta$ and IL-6) and inflammatory enzymes (COX-2 and 5-LOX), and upregulation of the anti-inflammatory cytokine IL-10. However, further research should be performed to clarify the mechanisms of the anti-arthritic activity of PF.

\section{References}

1. Bevaart L, Vervoordeldonk MJ and Tak PP: Collagen-induced arthritis in mice. Methods Mol Biol 602: 181-192, 2010.

2. Mclnnes IB and Schett G: The pathogenesis of rheumatoid arthritis. N Engl J Med 365: 2205-2219, 2011.

3. Firestein GS: Evolving concepts of rheumatoid arthritis. Nature 423: 356-361, 2003.

4. Lima-Garcia JF, Dutra RC, da Silva K, Motta EM, Campos MM and Calixto JB: The precursor of resolving D series and aspirin-triggered resolving D1 display anti-hyperalgesic properties in adjuvant-induced arthritis in rats. Br J Pharmacol 164: 278-293, 2011.

5. Momohara S, Kawakami K, Lwamoto T, Yano K, Sakuma Y, Hiroshima R, Imamura H, Masuda I, Tokita A and Ikari K: Prosthetic joint infection after total hip or knee arthroplasty in rheumatoid arthritis patients treated with nonbiologic and biologic disease-modifying antirheumatic drugs. Mod Rheumatol 21: 469-475, 2011.

6. Pincus $\mathrm{T}$ and Cutolo $\mathrm{M}$ : Clinical trials documenting the efficacy of low-dose glucocorticoids in rheumatoid arthritis. Neuroimmunomodulation 22: 46-50, 2015.

7. Atzeni F, Benucci M, Sallì S, Bongiovanni S, Boccassini L and Sarzi-Puttini P: Different effects of biological drugs in rheumatoid arthritis. Autoimmun Rev 12: 575-579, 2013.

8. Kaito T, Hosono N, Ohshima S, Ohwaki H, Takenaka S, Fujiwara H, Makino T and Yonenobu K: Effect of biological agents on cervical spine lesions in rheumatoid arthritis. Spine (Phila Pa 1976) 37: 1742-1746, 2012.

9. Scott DL, Shipley M, Dawson A, Edwards S, Symmons DP and Woolf AD: The clinical management of rheumatoid arthritis and osteoarthsitis: Strategies for improving clinical effectiveness. $\mathrm{Br}$ J Rheumatol 37: 546-554, 1998.

10. Silman AJ and Hochberg MC: Epidemiology of the Rheumatic Diseases. 2nd edition. Oxford University Press, Oxford, 2001.

11. Watanabe T, Tanigawa T, Shiba M, Nadatani Y, Nagami Y, Sugimori S, Yamagami H, Watanabe K, Tominaga K, Fujiwara Y, et al: Anti-tumor necrosis factor agents reduce non-steroidal anti-inflammatory drug-induced small bowel injury in rheumatoid arthritis patients. Gut 63: 409-414, 2014

12. Dixon WG, Kezouh A, Bernatsky S and Suissa S: The influence of systemic glucocorticoid therapy upon the risk of non-serious infection in older patients with rheumatoid arthritis: A nested case-control study. Ann Rheum Dis 70: 956-960, 2011.

13. Ma Q, Chen G, Zhang J, Feng B and Xu X: Relationship of tetramethylpyrazine on expression of vascular endothelial growth factor and development of adjuvant-induced arthritis in rats. Zhongguo Zhong Yao Za Zhi 34: 2799-2802, 2009 (In Chinese).

14. Wang Y, Fang YF, Zhou X and Zhong B: Effect of different concentration sinomenine on cytokines mRNA expression of synoviocytes in rats. Zhong Guo Zhong Yi Yao Xin Xi Za Zhi 10: 25-27, 2003 (In Chinese).

15. Yin W, Wang TS, Yin FZ and Cai BC: Analgesic and anti-inflammatory prosperties of brueine and brueine $\mathrm{N}$-oxide extracted from seeds of Strychnos nux-vomica. J Ethnopharmacol 88: 205-214, 2003. 
16. Xiao C, He YH, Huang FH, Lu C, Zhao LH, Zhao HY, Zhou J, Teng JR and Lu AP: Influence of tripterygium polyglycoside on free radicals and inflammatory factors in rats with adjuvant arthritis. Beijing Zhong Yi Yao Da Xue Xue Bao 29: 389-392, 2006 (In Chinese).

17. Zhu L, Wei W and Zheng YQ: Effect and mechanism of action of total glucosides of paeony on synoviocytes from rats with collagen-induced arthritis. Yao Xue Xue Bao 41: 166-170, 2006 (In Chinese)

18. Liu M, Dai Y, Yao X, Li Y, Luo Y, Xia Y and Gong ZN: Anti-rheumatoid arthritic effect of madecassoside on type II collagen induced arthritis in mice. Int Immunopharmcol 8: 1561-1566, 2008

19. Chen XG, Song XF, Cui XJ, Pan L and Xiao Q: The experimental research on the expression of Fas/FasL and Bcl-2/Bax correlated with apoptosis in synoviocyte of adjuvant arthritis with artesunate. Shi Zhen Guo Yi Guo Yao 17: 1926-1928, 2006 (In Chinese).

20. Cuzzocrea S, Saadat F, Di Paola R and Mirshafiey A: Artemether: A new therapeutic strategy in experimental rheumatoid arthdtis. Immunopharmacol Immunotoxicol 27: 615-630, 2005.

21. Dai Q, Fang J and Zhang FS: Dual role of shikonin in early and late stages of collagen type II arthritis. Mol Biol Rep 36: 1597-1604, 2009.

22. Ding F, Zhuang Y and Chang Y: Effects of Sanwushaoxie decoction on IL-18, TNF-alpha, IL-4 and IL-10 in patients with rheumatoid arthritis. Zhongguo Zhong Yao Za Zhi 35: 790-793, 2010 (In Chinese)

23. Huang Y, Zhou XP, Wang MY, Zhou LL, Yang YH, Shen JR and $\mathrm{Lu}$ Y: Effects of Qingluo Tongbi Granula on proliferation of fibroblast-like synoviocytes and RANKL expression in rheumatoid arthritis patients. Zhong Yao Xin Yao Yu Lin Chuang Yao Li 20: 510-512, 2009 (In Chinese).

24. Zhang C, Li MX, Shang XF, He XR and Jia ZP: Analgesic and anti-inflammatory activities of Phlomis younghusbandii. Hua X Yao Xue Za Zhi 26: 135-137, 2011 (In Chinese).

25. Gao YL, Lin RC, Wang GL, Zhao HR, Gao Y and Bianba CR: HPLC fingerprint of Phlomis younghusbandii. Zhong Yao Cai 30: 919-922, 2007 (In Chinese).

26. Li M, Zhang C, Wei L, Fan P, Zhang Q and Jia Z: Determination of five iridoid glycosides in Phlomis younghusbandii by HPLC. Zhongguo Zhong Yao Za Zhi 36: 594-597, 2011 (In Chinese).

27. Wang QS, Yang L, Cui WY, Chen L and Jiang YH: Anti-inflammatory and anti-nociceptive activities of methanol extract from aerial part of Phlomis younghusbandii Mukerjee. PLos One 9: e89149, 2014.

28. Liu QY, Sun B, Yang SY, Lv L and Liu Y: Therapeutic effect of radix Achyranthis bidentatae guiding function on diclofenac sodium induced adjuvant rats arthritis. Trad Chin Drug Res Clin Pharmacol 20: 408-411, 2009.

29. Feng F, Ding ZJ and Liu J: The study on the model of adjuvant arthritis in rats. Tian Jin Yao Xue 16: 1-4, 2004 (In Chinese).

30. Perera PK, Peng C, Xue L, Li Y and Han C: Ex vivo effect of Chinese herbal pill Yi Shen Juan Bi (YJB) on experimental arthritis. J Ethnopharmacol 134: 171-175, 2011.

31. Sindhu G, Ratheesh M, Shyni GL, Nambisan B and Helen A: Anti-inflammatory and antioxidative effects of mucilage of Trigonella foenum graecum (Fenugreek) on adjuvant induced arthritic rats. Int Immunopharmacol 12: 205-211, 2012.

32. Kokkola R, Li J, Sundberg E, Aveberger AC, Palmblad K, Yang H, Tracey KJ, Andersson U and Harris HE: Successful treatment of collagen-induced arthritis in mice and rats by targeting extracellular high mobility group box chromosomal protein 1 activity. Arthritis Rheum 48: 2052-2058, 2003

33. Pu J, Fang FF, Li XQ, Shu ZH, Jiang YP, Han T, Peng W and Zheng CJ: Matrine exerts a strong anti-arthritic effect on type II collagen-induced arthritis in rats by inhibiting inflammatory responses. Int J Mol Sci 17: 1410, 2016.
34. Sundaram R, Naresh R, Shanthi P and Sachdanandam P. Antihyperglycemic effect of iridoid glucoside, isolated from the leaves of Vitex negundo in streptozotocin-induced diabetic rats with special reference to glycoprotein components. Phytomedicine 19: 211-216, 2011.

35. Zhang LL, Wei W, Yan SX, Hu XY and Sun WY: Therapeutic effects of glucosides of Cheanomeles speciosa on collagen-induced arthritis in mice. Acta Pharmacol Sin 25: $1495-1501,2004$

36. Zheng CJ, Zhao XX, Ai HW, Lin B, Han T, Jiang YP, Xing X and Qin LP: Therapeutic effects of standardized Vitex negundo seeds extract on complete Freund's adjuvant induced arthritis in rats. Phytomedicine 21: 838-846, 2014.

37. Anderson GD, Hauser SD, McGarity KL, Bremer ME, Isakson PC and Gregory SA: Selective inhibition of cyclooxygenase (COX)-2 reverses inflammation and expression of COX-2 and interleukin 6 in rat adjuvant arthritis. J Clin Invest 97: 2672-2679, 1996

38. Radhika A, Jacob SS and Sudhakaran PR: Influence of oxidatively modified LDL on monocyte-macrophage differentiation. Mol Cell Biochem 305: 33-43, 2007.

39. Zhao B, Liang HX, Yu YF and Dong XP: A new furanolabdane diterpene glycoside from Phlomis younghusbandii Mukerjee. Acta Pharm Sin 44: 60-62, 2009.

40. Muhammad N, Saeed M, Gilani SN, Hap IU and Khan H: Analgesic and anti-inflammatory profile of n-hexane fraction of Viola betonicifolia. Trop J Pharm Res 11: 963-969, 2012.

41. Reddy SK, Kumar SA, Kumar VD and Ganapaty S: Anti-inflammatory and analgesic activities of Amorphallus bulbifer (Roxb) Kunth whole plant. Trop J Pharm Res 11: 971-976, 2012.

42. Kremer JM, Biqauoette J, Michalek AV, Timchalk MA Lininger L, Rynes RI, Huyck C,Zieminski J and Bartholomew LE: Effects of manipulation of dietary fatty acids on clinical manifestations of rheumatoid arthritis. Lancet 325: 184-187, 1985.

43. Lin B, Zhao Y, Han P, Yue W, Ma XQ, Rahman K, Zheng CJ, Qin LP and Han T: Anti-arthritic activity of Xanthium strumarium L. extract on complete Freund's adjuvant induced arthritis in rats. J Ethnopharmacol 155: 248-255, 2014.

44. Chen JY, Wu H, Li H, Hu SL, Dai MM and Chen J: Anti-inflammatory effects and pharmacokinetics study of geniposide on rats with adjuvant arthritis. Int Immunopharmacol 24: $102-109,2015$.

45. Tracey KJ, Vlassara H and Cerami A: Cachectin/tumor necrosis factor. Lancet 1: 1122-1126, 1989.

46. Boissier MC, Semerano L, Challal S, Saidenberg-Kermanach N and Falqarone G: Rheumatoid arthritis: From autoimmunity to synovitis and joint destruction. J Autoimmun 39: 222-228, 2012.

47. Carteron NL: Cytokines in rheumatoid arthritis: Trials and tribulations. Mol Med Today 6: 315-323, 2000.

48. Pablos Álvarez JL: Interleukin 6 in the physiopathology of rheumatoid arthritis. Reumatol Clin 5: 34-39, 2009.

49. Bozukurt FY, Yetkin Ay Z, Berker E, Tepe E and Akkus S: Anti-inflammatory cytokines in gingival crevicular fluid in patients with periodontitis and rheumatoid arthritis: A preliminary report. Cytokine 35: 180-185, 2006.

50. Crofford LJ: COX-1 and COX-2 tissue expression: Implications and predictions. J Rheumatol 49: 15-19, 1997.

51. Bombardieri S, Cattani P, Ciabattoni G, Di Munno O, Pasero G, Patrono C, Pinca E and Pugliese F: The synovial prostaglandin system in chronic inflammatory arthritis: Differential effects of steroidal and nonsteroidal anti-inflammatory drugs. Brit J Pharmacol 73: 893-901, 1981

52. Charlier $\mathrm{C}$ and Michaux C: Dual inhibition of cyclooxygenase-2 (COX-2) and 5-lipoxygenase (5-LOX) as a new strategy to provide safer non-steroidal anti-inflammatory drugs. Eur J Med Chem 38: 645-659, 2003. 